\title{
Recommendation Model Using User Reviews and Deep Learning Approach
}

\author{
Nyein Ei Ei Kyaw ${ }^{1}$ and Thinn Thinn Wai ${ }^{2+}$ \\ ${ }^{12}$ University of Information Technology, Yangon, Myanmar
}

\begin{abstract}
Due to the growing amount of information on the web, the information overload problem arises for users to discover relevant products easily and quickly. Collaborative Filtering $(\mathrm{CF})$ is a recommender system which uses the user-item rating matrix to make suggestions to users by finding many users' similar preferences. User reviews have information about products and users' opinions than ratings. Using Opinionbased ratings in the rating prediction process of recommender system can reflect the detail preference of users effectively and accurately. In this paper, sentiment analysis is used to find the opinions of the user and matrix factorization that applied neural network is used to predict the ratings for the recommendation. The experiment is performed on the dataset of the hotel reviews and rating prediction accuracy is evaluated by measuring the prediction error called the Root Mean Square Error (RMSE).
\end{abstract}

Keywords: Collaborative Filtering, Opinion-based ratings, sentiment analysis, neural network

\section{Introduction}

Nowadays, many online resources, including e-commerce platforms and social media have been widely applied recommendation system to tackle the issue of information overload. Recommendation systems are intelligent systems that address the information overload problem by using information retrieval and classification methods, and later by using deep learning-based methods.

Collaborative filtering (CF) approaches are commonly used in many electronic commerce applications, such as Amazon, Twitter, and Google News, in the recommendation process. Collaborative filtering does not required profile data, it only relies on observed user behavior for making recommendations. CF predicts a user's preference for items by finding similarities using the preference history of several other users. CF can be either memory-based methods or model-based methods. Memory-based methods predict the user's unknown preferences based on other similar users or items. Model-based approaches predict the unknown preference of the user by using the machine learning algorithm and data mining algorithms. Since the traditional $\mathrm{CF}$ uses the user-item rating matrix which represents a numerical rating only, there are many disadvantages, such as issue with data sparsity and new user problems. The data problem arises when the user doesn't give a rating on every item. In addition, ratings cannot capture the detailed users' interest. Matrix factorization (MF) is model based CF method which estimates ratings by decomposing the user-item matrix which represents user ratings or preferences of user into two low dimensional matrix as user latent factors and item latent factors and then performing the inner dot product of these two latent factors. The model then learns the two low dimensional space to minimize the loss function. User and item relationships are modeled in the same latent spaces and rating scores are calculated using linear operation, the inner dot product. The drawback of matrix factorization is that it can only represent a new user in a same latent space concerning all other user representations.

Various areas such as computer vision, audio recognition and natural language processing have been applied deep learning approaches due to the powerful representation learning abilities. Deep learning is also employed in the recommendation process to improve the quality of recommender system. Deep learning techniques are used to solve the challenges of recommender system such as scalability and sparsity. It also used in recommender systems to model either user-item preference matrix or auxiliary information, such as a textual description of items, audio feature of musics and visual content of the image.

\footnotetext{
+ Corresponding author. Tel.: Tel.: + 959-792104511; fax: +951-664250.
}

E-mail address: nyeineieikyaw@uit.edu.mm 
Recently, many researches tried to apply review text in the recommendation process to improve the prediction accuracy of recommender system. A review based recommender systems use information from the review text such as items' properties or user opinions extracted from the sentiment analysis process. Today's, customer reviews become an important role to find specific preferences by finding their opinions through online products' reviews, blogs and social networking sites. Therefore, this paper propose the recommendation model that applied the sentiment analysis process to represent the user preference as opinion score based ratings in user-item rating matrix and then neural network based matrix factorization approach for learning the complex relationships and latent spaces between users and items in the rating prediction process of recommendation system.

The rest of the paper is structured as follows. Section 2 presents the related work concerned with the proposed system. Section 3 gives an architecture of the proposed model. Section 4 discusses the experimental results of the proposed model in detail. Section 5 presents the conclusion.

\section{Related Work}

There are two research categories related to the proposed system. The first one is sentiment analysis based recommender system and the second one is deep learning-based recommender system. A variety of sentiment analysis based recommendation methods have been introduced to improve the efficiency of the recommender systems In paper[1], a multi-criteria recommendation method was proposed using a neighborhood-based CF algorithm that applies multi-faceted representation of user preference to predict the target user's interest on a specific product to meet the different user requirements. In the sentiment analysis process, features are extracted by using general corpus (British National Corpus BNC) and restaurant domain corpus. Model-based sentiment analysis algorithms are used to extract sentiment scores. The ratings prediction is performed by using the weighted summation approach with the top k neighbors of the user.

In order to overcome the constraints of the traditional collaborative algorithm, the authors of the paper [2] developed an item-based collaborative filtering method with the sentiment analysis approach. Aspect dictionary is constructed by using a bag of word to extract features and the logistic regression method is used to extract sentiment scores and then the item-based collaborative filtering approach is used to recommend top item lists to users. In paper [3], a personalized recommendation model based on topic modeling was proposed. The preference of users is obtained by extracting the topic of each review using the Latent Dirichlet Allocation (LDA). A new matrix is proposed to measure the similarity among users that alleviates the CF method's sparsity problem.

Recently, deep learning methods have been applied in the recommendation process to obtain more reliable and accurate results. In paper [4], deep matrix factorization model with neural network architecture has been applied in the recommendation process. The proposed model learns a specific low-dimensional space for user and item representations by taking into account both explicit ratings and implicit feedback to obtain better recommendation results. Another paper work [5] proposed the Deep Cooperative Neural Networks (DeepCoNN) model which also uses feedback from reviews to learn user behaviors and properties of items. The rating is calculated by learning hidden latent factors for users and items. The convolutional neural networks $(\mathrm{CNN})$ are trained together to get the lowest prediction error. Paper [6] proposed a deep neural network for learning non-linear interaction function rather than the inner product of matrix factorization by using implicit feedback. They extract the interaction status (one or zero) of user on items to represent the relationship between users and items. They learn the non-linear interaction function by using users' interaction status.

The related papers for both sentiment analysis based recommender systems mentioned above are applied the traditional collaborative filtering approaches and deep learning based recommender system are utilized the rating scores and properties of items for recommendation process. In this paper, the proposed system developed the deep learning based recommendation model which considers user opinions extracted from reviews. Sentiment analysis approach is used to find out the user's specific opinions for representing the relationship of user and item as opinion based rating. Then, neural network architecture is applied in rating prediction process to learn the complex interactions among users and items. 


\section{Proposed System Architecture}

In traditional collaborative filtering recommendation approach, user behavior is represented by users' historical ratings, which is usually expressed as a rating matrix. In this paper, user behavior is expressed by the opinion based rating extracted from the sentiment analysis process to define rating matrix R. Rating matrix R consists the rating score $r_{i j}$ where $r_{i j}$ can be either opinion based ratings or zero if user doesn't write reviews about the item. One of the problems of recommender system is to predict the rating of each unobserved entries $r_{i j}$ in $\mathrm{R}$ which is used for ranking the products. This system predicts the rating scores which expressed users' opinions by combining the lexicon-based sentiment analysis process and neural network based rating prediction process.

At the sentiment analysis process, user opinion scores are extracted from users' reviews by using the SentiWordNet lexicon. The opinion scores extracted from the lexicon is in the interval zero and one. Therefore the continuous opinion scores are discretized into distinct rating value range 1 if score is between 0.0 and $0.1,2$ if score is between 0.2 to $0.4,3$ if score is between 0.4 to $0.6,4$ if score is between 0.6 and 0.8 and 5 if score is between 0.8 and 1.0 by equal binning. After that, the neural network based rating prediction process is performed by using these resulted opinion based ratings. The overview of the proposed model is shown in figure 1.

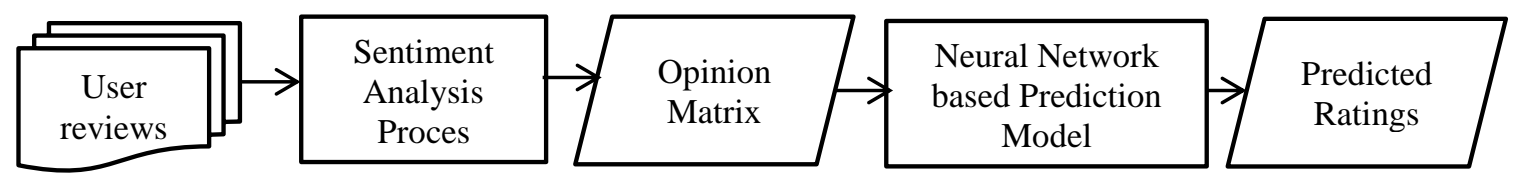

Fig 1: Architecture of the proposed system

\subsection{Sentiment Analysis}

The sentiment analysis process aims to find user opinions from reviews by extracting the sentiment scores to represent the user-item relationships. In this process, there are four sub-processes: preprocessing, aspect extraction, opinion words extraction, and sentiment scores extraction.

- Preprocessing: The input reviews are preprocessed to reduce unnecessary data in the process of sentiment analysis. Firstly, the review documents are tokenized into single words. Irrelevant symbols such as digits, punctuation marks, and stop words such as "a, and, the," etc., were omitted from the dataset, which are not required for the phase of sentiment analysis. To get the base or dictionary form of a word, the resulting terms are lemmatized, for example, terms like "feeling" are translated into "feel," etc. The lemmatized words are then marked with the NLTK POS tagger. The resulting POS tagged words are the eight parts of the speech, such as adverb, verb, noun, adjective etc.

- Aspect extraction: This process extracts the aspects of the item listed in the reviews. Aspects are noun to define the item's attributes, such as location, place, food, etc. The most frequent noun is chosen as aspects.

- Opinion words extraction: It is the process of extracting the opinion words to find review's sentiment scores. Words such as an adjective, adverb, and verb near the stated features are extracted as opinion words.

- Sentiment scores extraction: Sentiment score extraction is performed by using the SentiWordNet lexicon. The resulting opinion words are used to find the sentiment scores. Firstly, the score of the opinion word is extracted from the SentiwordNet lexicon. Sentence score is the average score of opinion words in the sentence. The overall opinion score of each review is the average score of sentences in the review.

\subsection{Rating Prediction Model}

The rating prediction model, the matrix factorization, implemented as neural network is used to learn complex interactions between users and items to predict unobserved ratings. The neural network has three layers: an input layer, a hidden layer, and one output layer. In this system, the input layer accepts the users' opinion based ratings matrix. The ratings are projected into a user latent vector and an item latent vector by using embedding layer. The resulted user and item latent vectors are then fed into the hidden layer to 
discover the user item relationship by performing element wise product of two vectors. The final output layer produces the predicted ratings by using the sigmoid activation function. Figure 2 shows the system flow of the rating prediction model.

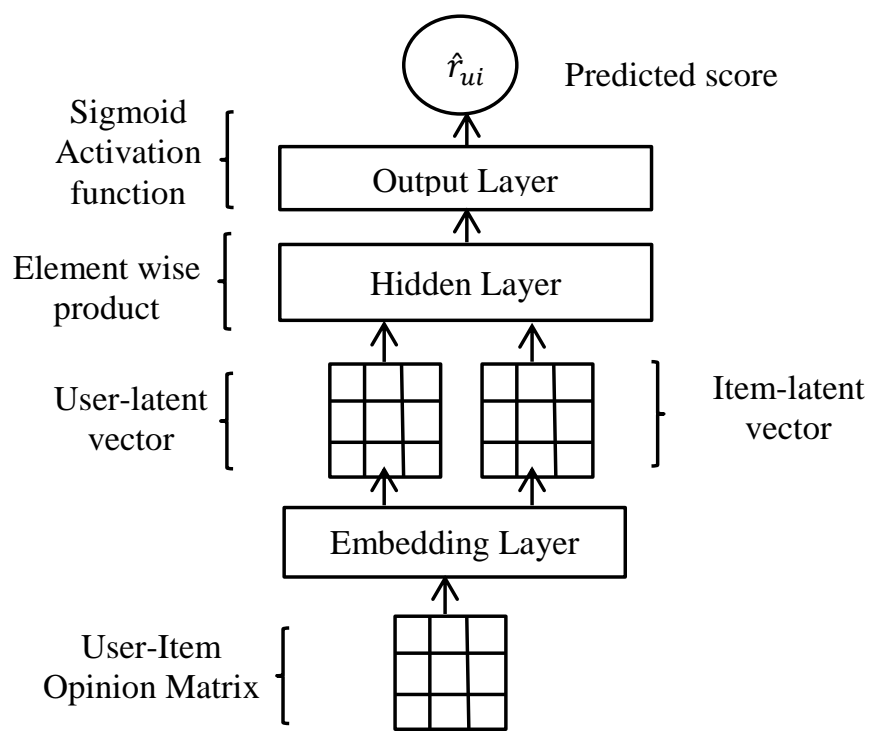

Fig 2: Rating prediction model

In the proposed rating prediction model, the unobserved ratings are predicted by using the following equation 1 .

$$
\widehat{r_{u l}}=f\left(P, Q \mid \theta_{f}\right)
$$

$P \in R^{M \times K}$ and $Q \in R^{N \times K}$ is the latent vector of users and items.

$\theta_{f}$ is the model parameter of the mapping function $\mathrm{f}$.

The mapping function $\mathrm{f}$ of hidden layer can be defined as the element wise product of a user latent vector and an item latent vector with the user bias $b_{u}$ which is the average rating of user $u$ and item bias $b_{i}$ which is the average rating of item $i$ with the following equation 2.

$$
f_{1}(P, Q)=P . Q+b_{u}+b_{i}
$$

The output layer produces the predicted rating by using the nonlinear activation function, sigmoid $\sigma$ and weight matrix $\mathrm{W}^{\mathrm{T}}$. It accepts the result from the hidden layer and multiply the weight matrix and then add user bias and item bias with the following equation 3 .

$$
\hat{r}_{u i=} \sigma\left(W^{T} f_{1}+b_{u}+b_{i}\right)
$$

\section{Experimental Results}

As for the evaluation of the system, Kaggle hotel review dataset [7] given by Datafiniti's Business Database is used. It includes about 1000 hotels and their reviews, as well as related information such as hotel location, name, rating, review data, title, username and more. This research is tested using Google Colab, which is a free cloud service to develop deep learning applications, python 3.5.3, Tensorflow 1.15 .0 and NLTK toolkit.

The training process is performed on the $80 \%$ data as training data and $20 \%$ as testing data. The proposed model is trained using 50 epochs, 5 batch size, learning rate is 0.001 , L2 regularization and Adam optimizer. The performance of the model is measured by comparing the results of the proposed model with basic matrix factorization in term of Root Mean Squared Error (RMSE) as shown in equation 4.The lower RMSE values indicate better prediction accuracy.The two model use latent features vector size, 10. According to the results, the RMSE for the testing dataset of neural network based matrix factorization has less than 0.010 and basic matrix factorization has 1.36 respectively. The RMSE for training set of neural network based matrix factorization is 0.015 and less than 1.28 for training dataset. Therefore, the proposed 
model gets more accurate prediction results than basic MF. The evaluation results are shown in figure 3 (a) and (b).

$$
R M S E=\sqrt{\frac{\sum_{i=1}^{n}\left(r_{u i-\widehat{r}_{u i}}\right)^{2}}{n}}
$$

$r_{u i}$ is the actual rating of user $\mathrm{u}$ on item $\mathrm{i}$.

$\hat{r}_{u i}$ is the predicted rating of user $u$ on item $i$.

$\mathrm{n}$ is the number of ratings.

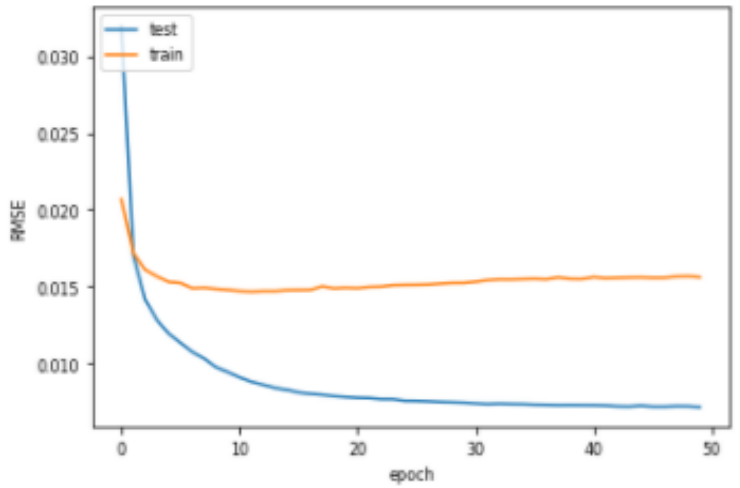

(a)

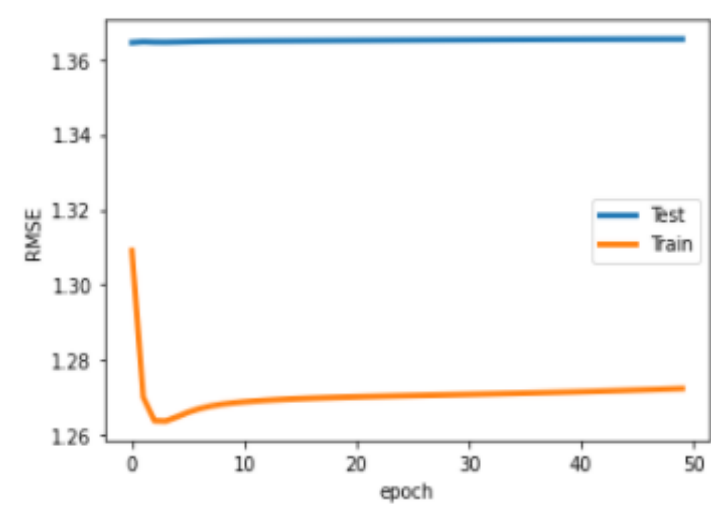

(b)

Fig. 3: Evaluation results of Neural network based MF (a) and basic MF (b)

\section{Conclusion}

In recent year, user reviews are applied in recommender systems to make suggestions which consider users' opinions on products. This paper proposed the rating prediction model in collaborative filtering recommendation approach by combining sentiment analysis and neural network based matrix factorization to improve the performance of recommender system. The experiment results have shown that the accuracy of the rating prediction of neural network based matrix factorization can improve the basic MF. As a future work, evaluation has been performing on different datasets by applying other advanced sentiment analysis approach and more deeper network is utilized in rating prediction process.

\section{References}

[1] Musto C, de Gemmis M, Semeraro G, Lops P. A multi-criteria recommender system exploiting aspect-based sentiment analysis of users' reviews. In Proceedings of the eleventh ACM conference on recommender systems 2017 Aug 27 (pp. 321-325).

[2] Dubey A, Gupta A, Raturi N, Saxena P. Item-Based Collaborative Filtering Using Sentiment Analysis of User Reviews. International Conference on Application of Computing and Communication Technologies 2018 Mar 9 (pp. 77-87). Springer, Singapore.

[3] Xu J, Zheng X, Ding W. Personalized recommendation based on reviews and ratings alleviating the sparsity problem of collaborative filtering. In2012 IEEE Ninth International Conference on e-Business Engineering 2012 Sep 9 (pp. 9-16). IEEE.

[4] Xue HJ, Dai X, Zhang J, Huang S, Chen J. Deep Matrix Factorization Models for Recommender Systems. In IJCAI 2017 Aug 19 (Vol. 17, pp. 3203-3209).

[5] Zheng L, Noroozi V, Yu PS. Joint deep modeling of users and items using reviews for recommendations. In Proceedings of the Tenth ACM International Conference on Web Search and Data Mining 2017 Feb 2 (pp. 425434).

[6] He X, Liao L, Zhang H, Nie L, Hu X, Chua TS. Neural collaborative filtering. In Proceedings of the 26th international conference on world wide web 2017 Apr 3 (pp. 173-182).

[7] https://www.kaggle.com/datafiniti/hotel-reviews. 